\title{
MINIMAL INDEX TORSION-FREE SUBGROUPS OF KLEINIAN GROUPS
}

\author{
KERRY N. JONES \\ ALAN W. REID
}

\begin{abstract}
It was shown by Edmonds, Ewing and Kulkarni in 1982 that the minimal index of a torsion-free subgroup of a finitely generated Fuchsian group of the first kind $G$ is bounded above by twice the LCM of the orders of the finite subgroups of $G$. Here we show that no such result is possible for Kleinian groups. Specifically, we exhibit a sequence $\Gamma_{k}$ of co-compact Kleinian groups for which the ratio of the minimum index to the LCM is arbitrarily large.

The construction of the $\Gamma_{k}$ uses generalized triangle groups and unknotting tunnels of 2-bridge knots. We also derive some results of independent interest involving these two constructs.
\end{abstract}

\section{Section 0 - Introduction}

A Kleinian group $\Gamma$ is a discrete subgroup of $\operatorname{PSL}(2, \mathbb{C})$, the full group of orientationpreserving isometries of 3-dimensional hyperbolic space. In the language of [T1] $Q=\mathbb{H}^{3} / \Gamma$ is a hyperbolic 3-orbifold; that is a metric 3-orbifold in which all sectional curvatures are -1 , and for which $\Gamma$ is the orbifold fundamental group (see [T1] for further details). A Fuchsian group is a discrete subgroup of $\operatorname{PSL}(2, \mathbb{R})$ and as such acts discontinuously on the hyperbolic plane. We define $\Gamma$ to be of finite co-volume (resp. co-area) if the volume (resp. area) of the quotient orbifold $Q$ is finite.

By Selberg's Lemma, if $\Gamma$ is a Kleinian (resp. Fuchsian) group of finite co-volume (resp. co-area) it contains a torsion-free subgroup of finite index. By definition any torsion-free subgroup cannot contain any finite subgroups of $\Gamma$, so the index must be a multiple of the lowest common multiple of all orders of finite subgroups of $\Gamma$ (see [CFJR] for a proof). $\Gamma$ being of finite co-volume implies there are only finitely many conjugacy classes of finite subgroups. Thus, we make the following

1991 Mathematics Subject Classification. Primary: 57M50, Secondary: 30F40.

Key words and phrases. Kleinian group, torsion-free subgroup, generalized triangle group, 2-bridge link.

First author partially supported by Ball State University Office of Academic Research. The first author wishes to thank Rice University for hospitality during the time part of this research was conducted. Second author is supported by The Royal Society. The second author also wishes to thank C. Maclachlan for interesting discussions concerning generalized triangle groups. Both authors wish to thank J. Howie for helpful comments on an early version of this paper and the referee 
Definitions. Let $G$ be a finitely-generated group containing only finitely many conjugacy classes of finite subgroups and some torsion-free subgroup of finite index. Then, and

$\mathcal{L}(G)=$ the lowest common multiple of the orders of all finite subgroups of $G$

$\mathcal{M}(G)=$ the minimum index of a torsion-free subgroup of $G$.

Now non-trivial finite subgroups of a Fuchsian group are cyclic as follows from the structure theory of Fuchsian groups, and the only possibilities in the case of a Kleinian group are cyclic, dihedral, $A_{4}, S_{4}$ or $A_{5}$. In [EEK], it is shown that if $\Gamma$ is a Fuchsian group of finite co-area, then $\mathcal{M}(G) / \mathcal{L}(G) \leq 2$. Given that the structure of Fuchsian groups is well-understood, the proof of this theorem is remarkably subtle in the case where the underlying space of the quotient orbifold is the 2-sphere.

The corresponding question as to whether there exists a bound for $\mathcal{M}(G) / \mathcal{L}(G)$ in the case of Kleinian groups has been of some interest lately in the study of small volume hyperbolic 3-orbifolds. In particular if a bound exists, then this would, for example, allow for simplification in the proofs of [CFJR] in the identification of the smallest volume arithmetic hyperbolic 3-manifold. However, in [CFJR], a Kleinian group $\Gamma$ was constructed for which it was observed that $\mathcal{M}(\Gamma) / \mathcal{L}(\Gamma)=4$. The purpose of this paper is to prove

Theorem 1. For $k \in \mathbb{N}, k \geq 2$, there exists a Kleinian group $\Gamma_{k}$ such that $\mathcal{M}\left(\Gamma_{k}\right) / \mathcal{L}\left(\Gamma_{k}\right)>k$.

The construction of the $\Gamma_{k}$ involve Generalized Triangle Groups (defined below). These have attracted some attention recently for a variety of reasons (see for instance $[\mathrm{BMS}],[\mathrm{GL}]$, and [HMV]). An extension of our construction of the groups $\Gamma_{k}$ provides a partial answer to a question of M. Hagelberg in [G93] concerning which Generalized Triangle Groups can be the orbifold fundamental group of a compact orientable 3-orbifold (possibly with boundary). This question has been studied in special cases recently; see for example [H], [HMR] and [HMV].

\section{Section 1 - Generalized Triangle Groups and Tunnel Number 1 Links}

The construction of the Kleinian groups $\Gamma_{k}$ is closely connected with the notion of a Generalized Triangle Group, and tunnel number 1 links, which we now define:

Definition. A Generalized Triangle Group is a group admitting a presentation of the form

$$
\left\langle a, b: a^{m}, b^{n},(R(a, b))^{p}\right\rangle
$$

where $R(a, b)$ is any cyclically reduced element of the $Z_{m} * Z_{n}$ generated by a and $b$ which is not conjugate to a proper power and $m, n, p$ are integers greater than 1 .

This group will be denoted $\Delta(m, n, p, R(a, b))$.

Let $L$ be a knot or link in $S^{3}$. An unknotting tunnel for $L$ is an embedded arc $t$ in $S^{3}$ disjoint from $L$ with endpoints on $L$ such that the complement of a regular neighbourhood of $L \cup t$ in $S^{3}$ is a genus 2 handlebody. If an unknotting tunnel for $L$ exists, $L$ is called tunnel number 1 . Note if a link has tunnel number 1 it must have at most two components. Such a decomposition of the knot or link exterior leads to a 2-generator, 1-relator presentation of the group, the relator corresponding to a simple closed curve in the boundary of a genus 2 handlebody to which a 2-handle is attached. 


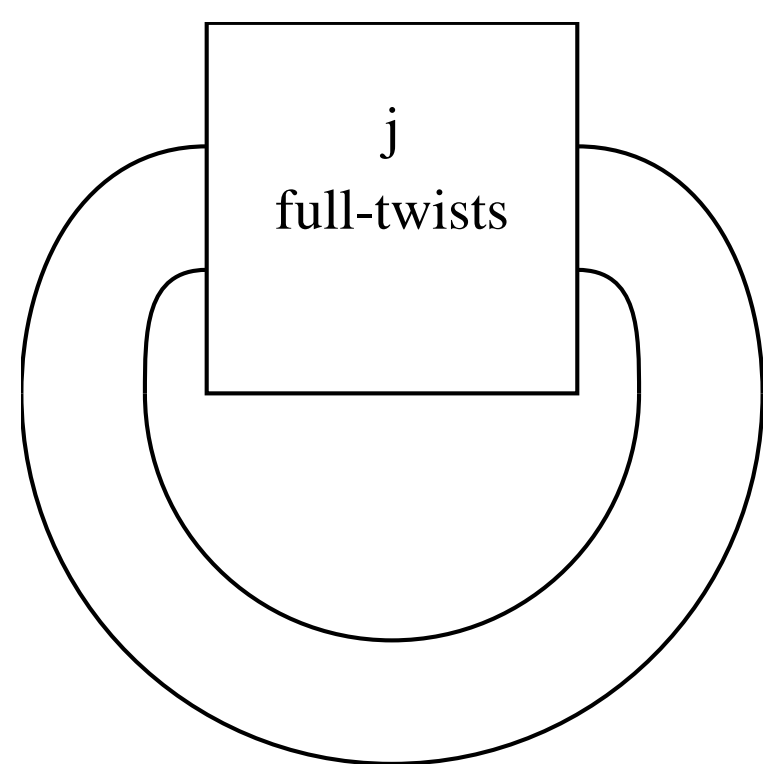

Figure 1

All 2-bridge knots and links are tunnel number 1: a pair of unknotting tunnels that are easy to see are the so-called "upper" or "lower" tunnels in a 4-plat projection of the knot or link. (cf. $[\mathrm{BM}]$ and $[\mathrm{AR}]$ for more on this). Each of these tunnel decompositions results in a 2-generator, 1-relator presentation for the fundamental group with a pair of meridians of the link as generators.

Let $m, n$ and $p$ be integers greater than 1. Form the labelled graph $\Sigma=$ $\Sigma(m, n, p)$ in $S^{3}$ as follows. Using the upper (or lower) tunnel $t$ above, each 2bridge knot or link yields a graph (with three edges and two vertices) which is then labelled by labelling the edges $m, n$ and $p$, indicating a cone angle of $2 \pi / i$ where $i$ is one of $\{m, n, p\}$ along the edge (we shall take $p$ to be the label of the tunnel). Let $Q=Q(t ; m, n, p)$ be the orbifold with these specifications as base and singular set, so that $Q$ is a compact orientable 3 -orbifold (strictly speaking, we must delete an open neighborhood of the vertices if the labelling of the incident edges is not spherical). It follows that $\pi_{1}^{\text {orb }}(Q)$ is of the form $\left\langle a, b: a^{m}=b^{n}=R(a, b)^{p}=1\right\rangle$. With this discussion we have:

\section{Lemma 2.}

$\pi_{1}^{\text {orb }}(Q)$ is a generalized triangle group.

We now discuss in a little more detail one family of orbifolds that we shall use below.

\section{Example}

For $j \geq 1$, let $T_{j}$ be the 2-bridge torus link with $j$ full-twists (see Figure 1 ), so $T_{1}$ is the Hopf link. Note that we may take either left or right twists here as convenient - we will simply get an orientation-reversal between the two and all orbifold fundamental groups we subsequently obtain will be isomorphic regardless of which orientation we choose.

Let $O_{j}$ be the orbifold obtained from $T_{j}$ as discussed above using the upper tunnel. Label the edges of the graph using the triple $\{3,3,2\}$ as shown in Figure 2 below.

\section{Lemma 3.}




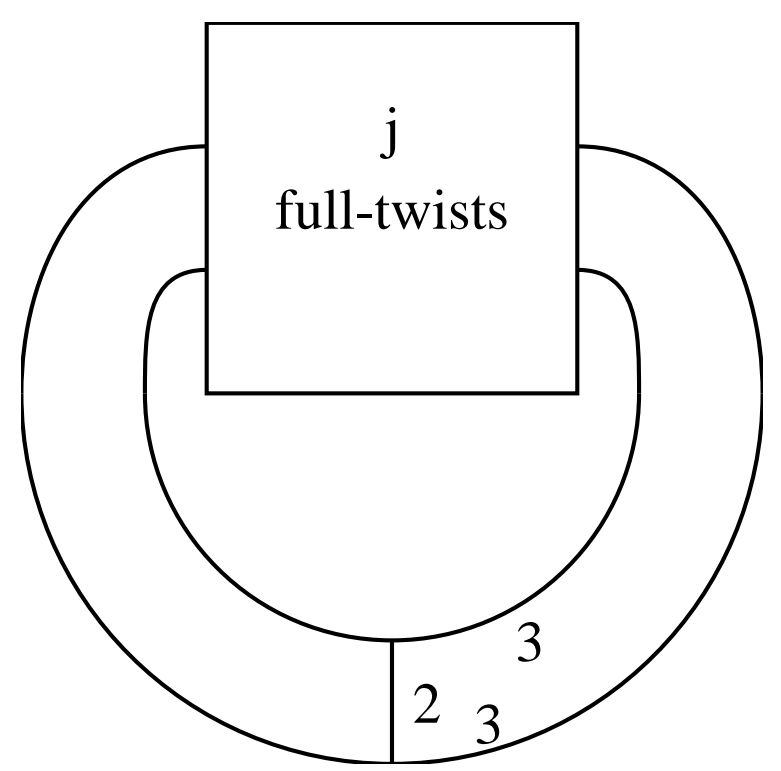

Figure 2

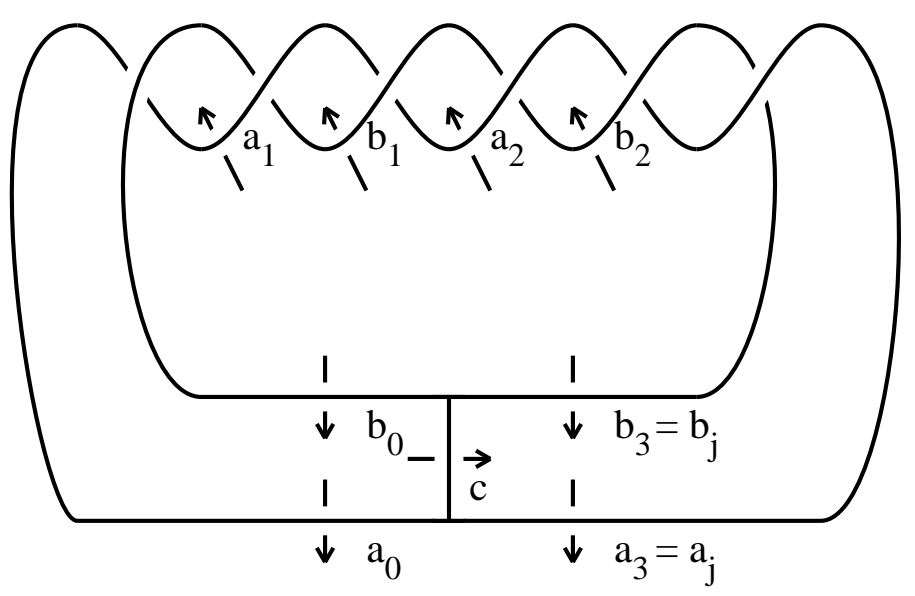

Figure 3

$\pi_{1}^{\text {orb }}\left(O_{j}\right)$ has a presentation $\left\langle a, b: a^{3}=b^{3}=\left((a b)^{j}\left(a^{-1} b^{-1}\right)^{j}\right)^{2}=1\right\rangle$.

Proof. We will use the Wirtinger presentation - refer to Figure 3, where the case $j=$ 3 is illustrated. The generators of $\pi_{1}^{\text {orb }}\left(O_{j}\right)$ are $c, a_{0}, a_{1}, \ldots, a_{j}, b_{0}, b_{1}, \ldots, b_{j}$. The relations come from three sources: torsion relations, vertex relations and crossing relations. The torsion relations are generated by $c^{2}=a_{0}^{3}=b_{0}^{3}=1$. The singular set vertices give the relations $c=b_{0}^{-1} b_{j}=a_{0} a_{j}^{-1}$. The crossings give relations

$$
\begin{aligned}
a_{i} & =b_{i-1} a_{i-1} b_{i-1}^{-1} \quad(i=1,2, \ldots, j) \\
b_{i} & =a_{i} b_{i-1} a_{i}^{-1}
\end{aligned}
$$

Repeatedly using these crossing relations to write all the $a_{i}$ and $b_{i}$ in terms of $a_{0}$ and $b_{0}$, we obtain

$$
\begin{aligned}
a_{i} & =\left(b_{0} a_{0}\right)^{i-1} b_{0} a_{0} b_{0}^{-1}\left(a_{0}^{-1} b_{0}^{-1}\right)^{i-1} \\
b_{i} & =\left(b_{0} a_{0}\right)^{i} b_{0}\left(a_{0}^{-1} b_{0}^{-1}\right)^{i}
\end{aligned}
$$


Substituting these into the vertex relations gives the single relation $c=\left(a_{0} b_{0}\right)^{j}\left(a_{0}^{-1} b_{0}^{-1}\right)^{j}$ so that

$$
\pi_{1}^{\text {orb }}\left(O_{j}\right)=\left\langle a_{0}, b_{0}: a_{0}^{3}=b_{0}^{3}=\left(\left(a_{0} b_{0}\right)^{j}\left(a_{0}^{-1} b_{0}^{-1}\right)^{j}\right)^{2}=1\right\rangle
$$

\section{Section 2 - The Construction}

In this section we construct the Kleinian groups $\Gamma_{k}$ advertised in Theorem 1 using the orbifolds $O_{j}$ given in the Example of $\S 1$.

Theorem 1 will be a consequence of the next two lemmas.

Lemma 4. For $j$ sufficiently large, $O_{j}$ is a closed hyperbolic 3-orbifold.

Proof.

Let $Q$ be the orbifold pictured in Figure 4. This is an orbifold whose base is the open solid torus.

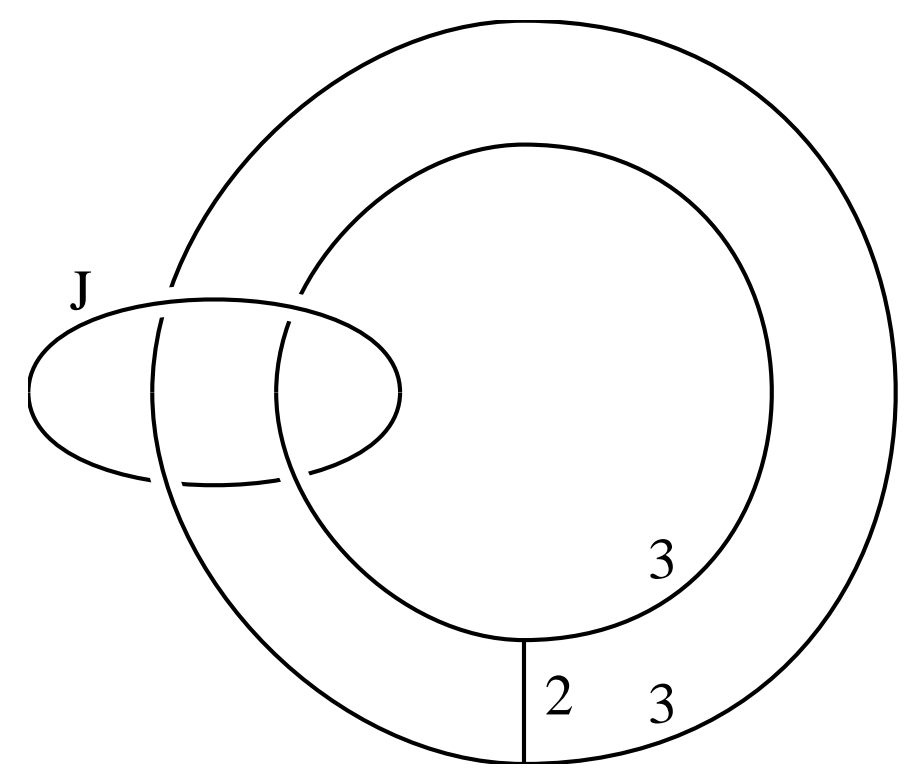

Figure 4

Notice that the orbifolds $O_{j}$ are obtained by $(1, j)$-Dehn surgery on the cusp of $Q$ corresponding to the unknotted component $J$ in Figure 4. We may also use $(-1, j)$-Dehn surgery and obtain an isomorphic fundamental group.

We shall show that $Q$ is hyperbolic and so Thurston's Hyperbolic Dehn Surgery Theorem implies the Lemma. A proof of the Dehn Surgery Theorem for orbifolds is given in $[\mathrm{DM}]$. To see that $Q$ is hyperbolic we proceed as follows. From $[\mathrm{FF}]$ for instance, the orbifold shown in Figure 5 is the hyperbolic orbifold $B$ arising as $\mathbb{H}^{3} / \operatorname{PSL}(2, \mathbb{Z}[\sqrt{-2}]) . \quad B$ is 2 -fold covered by $Q$, where the quotient map is given by the involution on the solid torus which rotates the solid torus about a core axis (the dashed curve in Figure 6).

These observations complete the proof.

In the case of $j=1$, the link in the singular set in this case is simply the Hopf Link, and the hence the labelled graph describing the singular set in this case is the Hopf link labelled 3 and the arc labelled 2. This orbifold appears in the list of spherical orbifolds in Table 8 of [Du1], so the group $G_{j}$ is finite and hence $O_{1}$ is 


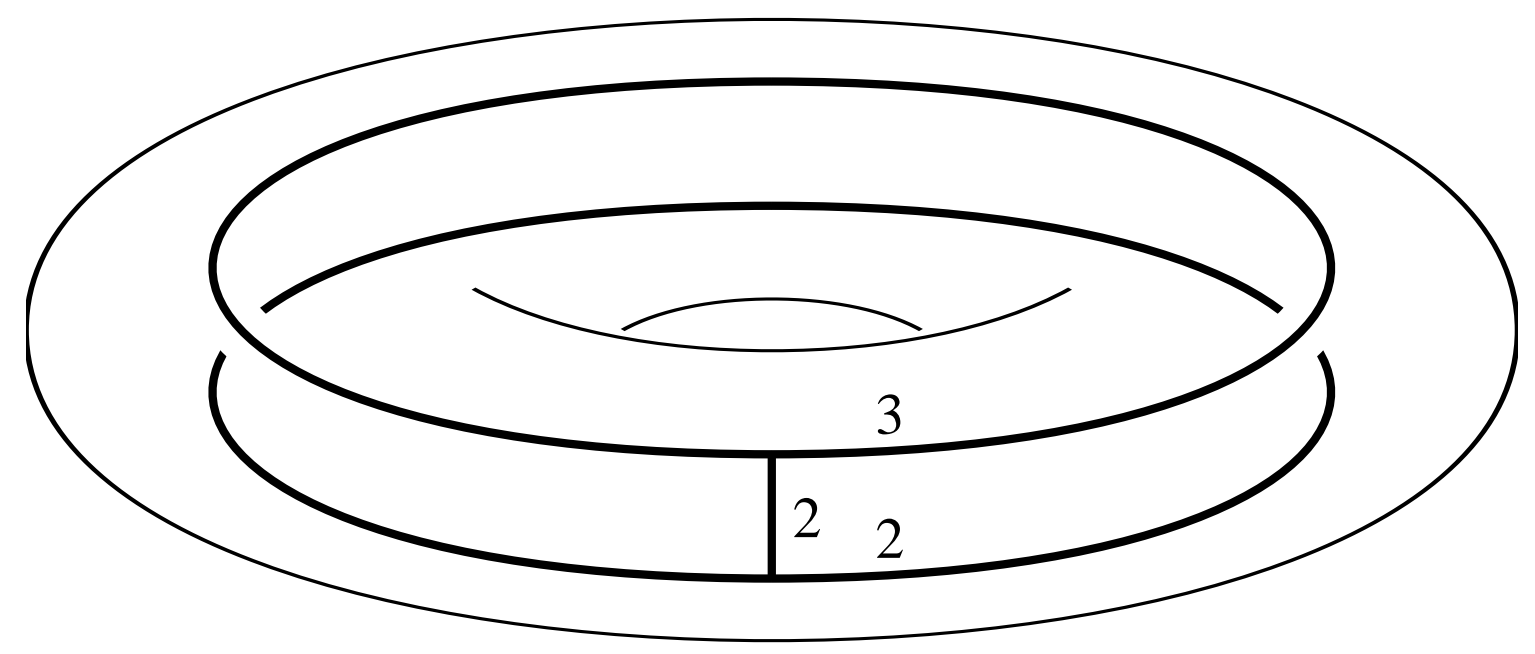

Figure 5

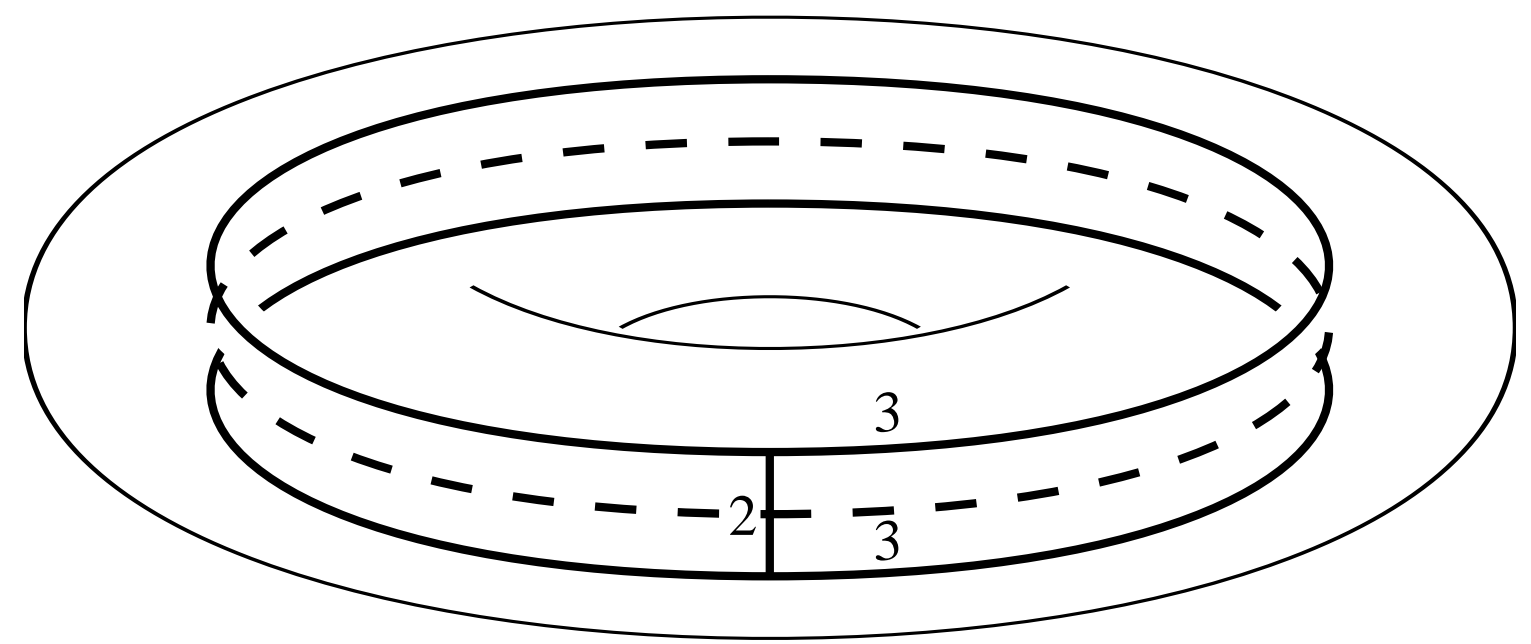

Figure 6

certainly not hyperbolic. In fact it has been shown in [LR] that this group has order 288. In $\S 3$, we shall see that the Thurston Orbifold Conjecture actually implies that $O_{j}$ is hyperbolic whenever $j>1$.

Define $\Gamma_{k}$ to be the Kleinian group which is the orbifold fundamental group of $O_{(12 k) !}$.

Lemma 5. $\mathcal{M}\left(\Gamma_{k}\right)>12 k$ for sufficiently large $k$.

Proof.

Suppose $H$ is a torsion-free subgroup of index $m \leq 12 k$. The permutation representation of $\Gamma_{k}$ on $H$ yields a homomorphism, $\varphi: \Gamma_{k} \rightarrow S_{m}$ such that $a$ and $b$ map to a product of disjoint 3 -cycles, and $(a b)^{(12 k) !}\left(a^{-1} b^{-1}\right)^{(12 k) !}$ maps to a 
product of 2-cycles. Note that we are making use of hyperbolicity to assert that all the relators actually have the order that they appear to have in the presentation. In $\S 3$, we shall see that this is actually independent of hyperbolicity, so that the hypothesis of "sufficiently large k" is really unnecessary here.

Since $S_{m}$ embeds as a subgroup of $S_{12 k}$ for all $m \leq 12 k$, we see that the order of any element of $S_{m}$ divides $(12 k)$ !. Hence $\varphi\left((a b)^{(12 k) !}\left(a^{-1} b^{-1}\right)^{(12 k) !}\right)=1$, since it is the product of two $(12 k)$ ! powers. Now the kernel of $\varphi$ is a subgroup of $H$, and this contradicts the assumption that $H$ is torsion-free.

All that remains to complete the proof of Theorem 1 is to note that for all $k$,

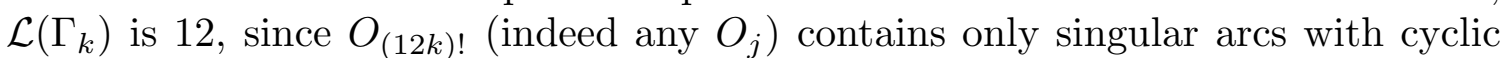
isotropy of order 2 and 3 and two singular vertices with $A_{4}$ isotropy. Thus, Lemma 5 implies Theorem 1.

It seems worthwhile noting that many "more obvious" constructions of orbifolds do not give examples where the conclusion of Theorem 1 is satisfied. Indeed for many cases, one can say exactly what the minimal index torsion-free subgroup is. These considerations, in part, led us to consider the examples above. The following is quite straightforward, not even requiring hyperbolicity.

Theorem 6. Let $M$ be a closed orientable 3-manifold, and $L=L_{1} \cup L_{2} \ldots \cup L_{n}$ a link embedded in $M$. Fix a framing $\left(\mathcal{M}_{i}, \ell_{i}\right)$ for each component. Let $Q$ denote the orbifold obtained by doing $\left(p_{1}, 0\right), \ldots\left(p_{n}, 0\right)$ orbifold Dehn surgery. Then $\pi_{1}^{\text {orb }}(Q)$ has a torsion free subgroup of index $\operatorname{LCM}\left(p_{1}, \ldots, p_{n}\right)$.

Proof.

This is merely an exercise in branched covers. Let $p_{j_{1}}, \ldots, p_{j_{m}}$ be the distinct prime factors of $\mathcal{L}=\operatorname{LCM}\left(p_{1}, \ldots, p_{n}\right)$. Using the framing given, there is a map from $\pi_{1}^{\text {orb }}(Q)$ to $\mathbb{Z}_{p_{j_{1}}} \oplus \ldots \oplus \mathbb{Z}_{p_{j_{m}}}$ obtained by factoring the map from $\pi_{1}(M \backslash L)$ through the homomorphism induced by the prescribed orbifold Dehn surgery. The kernel of this map is necessarily torsion-free.

The next result is somewhat more involved, but hyperbolicity is not needed here either. This result states that we cannot construct the orbifolds we desire by doing orbifold Dehn surgery on successively more complicated punctured surface bundles.

Theorem 7. Let $X$ be a surface bundle with fiber a surface of genus $g$ with $n \geq 1$ punctures. Frame each component of the boundary of $X$ as $\left(\mathcal{M}_{i}, \ell_{i}\right)$ where $\mathcal{M}_{i}$ is a component of the pre-image of a point in the fiber and $\ell_{i}$ is a boundary component of the fiber. Let $O$ be the orbifold obtained by $\left(m_{i} p_{i}, n_{i} p_{i}\right)$ orbifold Dehn surgery on component $i$ of $X$, where $m_{i}$ and $n_{i}$ are relatively prime and $n_{i}$ and $p_{i}$ are relatively prime. Let $G$ denote the orbifold fundamental group of $O$. Then, there is a constant $q$, depending only on $g, n$ and the $p_{i}$, such that $\mathcal{M}(G) / \mathcal{L}(G) \leq q$. In particular, $q$ is independent of the monodromy of $X$.

Proof. For the sake of clarity, denote the (free) fundamental group of the fiber of $X$ by $F$ and the mapping class group of $X$ by $M_{F}$.

First, consider the case in which all $m_{i}$ are 0 and all $n_{i}$ are 1 . The orbifold Dehn surgery in this case induces an orbifold structure on the fiber, which we denote by $O_{F}$ and its orbifold fundamental group by $G_{F}$. Furthermore, $G$ is an HNN-extension of $G_{F}$. Denote the HNN-extension element (some power of which represents all of the $\mathcal{M}_{i}$ ) by $t$. Now, $G_{F}$ has a torsion-free subgroup (of index at most $2 \mathcal{L}(G))$ which we denote by $H_{F}$. 
Next, we construct another subgroup, $K_{F}$ of $G_{F}$ which is also torsion-free and of finite index. We do this by pulling back $H_{F}$ to $F$, yielding a (free) subgroup $H_{F}^{\prime}$. Next, we observe that $M_{F}$ acts on $H_{F}^{\prime}$, so we intersect all conjugates of images of $H_{F}^{\prime}$ under the action of $M_{F}$, yielding a finite-index normal subgroup $K_{F}^{\prime}$. The key fact here is that $K_{F}^{\prime}$ is invariant under the action of $M_{F}$, hence the projection homomorphism from $F$ to $F / K_{F}^{\prime}$ is also invariant under the action of $M_{F}$. Let $K_{F}$ be the image of $K_{F}^{\prime}$ under the projection from $F$ to $G_{F}$. Since $K_{F}$ is torsion-free, the index of $K_{F}$ in $G_{F}$ is a multiple of $\mathcal{L}\left(G_{F}\right)$. So, let $r=\left[G_{F}: K_{F}\right]$ and let $q=r / \mathcal{L}(G)$. Now, there is a homomorphism $\varphi: G_{F} \rightarrow S_{r}$ which is the subgroup monodromy of $K_{F}$ (since $K_{F}$ is normal, it is in fact the projection map to $G_{F} / K_{F}$ embedded in $S_{r}$ ). As already observed, $\varphi$ is invariant under the action of $M_{F}$ on the generators of $G_{F}$ so that $\varphi$ extends trivially (by sending $t$ to the identity) to a homomorphism from $G$ to $S_{r}$. Abusing notation slightly, denote this homomorphism also by $\varphi$. So, the HNN-extension of $K_{F}$ by $t$ is a torsion-free subgroup of $G$ with index equal to $q \mathcal{L}(G)$. Thus, we have proven our result for the case in which $m_{i}=0$ and $n_{i}=1$.

For the more general case, we merely remark that the homomorphism $\varphi$ constructed above still works: since $K_{F}$ above is torsion-free, $\varphi\left(\ell_{i}\right)$ is a product of disjoint $p_{i}$-cycles. Furthermore, all of the $\mathcal{M}_{i}$ are sent to the identity by $\varphi$. So, the surgery curves are sent to $\varphi\left(\ell_{i}\right)^{n_{i}}$ which will still be a product of disjoint $p_{i}$-cycles, since $n_{i}$ and $p_{i}$ are relatively prime.

To actually calculate $q$ for a particular $\left(g, n,\left\{p_{i}\right\}\right)$ it suffices to go through the process outlined in the proof, which is rather tedious, but which could be easily automated. We have calculated that for $g=1, n=1, p_{1}=2$ (the simplest case) we have $q=4$ (the group $G_{F} / K_{F}$ is the order 8 group of unit quaternions).

\section{Section 3 - Geometric Structures for Generalized Triangle Groups}

In this section we extend our constructions in $\S 1$ and 2 to show that many generalized triangle groups have realizations as the orbifold fundamental groups of geometric 3 -orbifolds. This has been of some interest recently (see $[\mathrm{H}],[\mathrm{HMR}]$ and $[\mathrm{HMV}]$ ) and gives a partial answer to a question of Hagelberg in [G93]. Our methods of exhibiting a geometric structure use the Orbifold Conjecture of Thurston (see below).

We commence with a different description of the orbifolds obtained from 2-bridge knots or links and an unknotting tunnel discussed in $\S 2$.

Definition. Let $r, s$ be positive relatively prime integers with $r<s$ and $a, b$ be generators of a free group $G$. Then

$$
W(r, s, a, b)=\prod_{i=0}^{s-1} a^{(-1)^{[(2 i+1) r / s]}} b^{(-1)^{[(2 i+2) r / s]}}
$$

We will also abuse this notation slightly and use $W(r, s, a, b)$ where $a$ and $b$ are arbitrary elements of an arbitrary group to represent the obvious group element.

Theorem 8. Let $G=\Delta(m, n, p, R(a, b))$ be a Generalized Triangle Group and let $\rho: Z * Z \rightarrow Z_{m} * Z_{n}$ be the canonical homomorphism taking generators $(a, b)$ to generators $(c, d)$. Let $r, s$ be positive relatively prime integers with $r<s$ such that $\rho(R(a, b))$ (or its inverse) is the image of $W(r, s, c, d)$ under an automorphism 


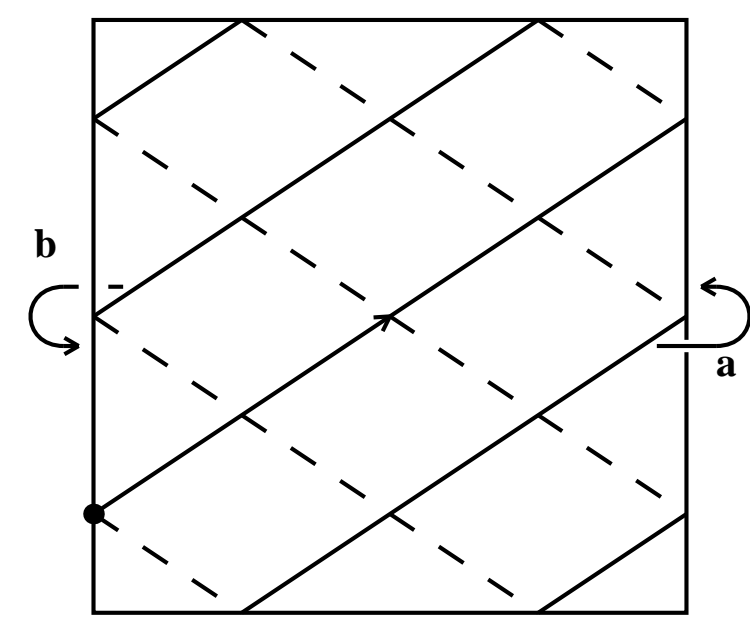

$\mathrm{W}(2,3, \mathrm{a}, \mathrm{b})=\mathrm{ab} \mathrm{b}^{-1} \mathrm{aba} \mathrm{a}^{-1} \mathrm{~b}$

Figure 7

of $Z_{m} * Z_{n}$. Then, $G$ is the orbifold fundamental group of a compact orientable 3-orbifold.

Proof. Assume that $G=\Delta(m, n, p, W(r, s, a, b))$ is a generalized triangle group with $r$ and $s$ as in the hypothesis. We shall construct a 3-orbifold whose fundamental group is isomorphic to $G$ by taking a genus-two handlebody $H$ (with fundamental group generated by $a$ and $b$ ) and adding singular 2-handles (with central singularities of order $m, n$ and $p$, respectively) along disjoint simple closed curves in the boundary representing $a, b$ and $W(r, s, a, b)$. The curves representing $a$ and $b$ may be taken to be the standard generating curves. Note that standard here means standard with respect to the usual handlebody picture. This standard handlebody will be mapped into $S^{3}$ in a very nonstandard way in general.

At this point, the only remaining difficulty is in ensuring that a simple closed curve representing $W(r, s, a, b)$ may be found in the boundary with the $a$ and $b$ curves removed. But this surface is a four-punctured sphere, and free homotopy classes of simple closed curves on a four-punctured sphere are represented by "lines of rational slope on a square pillowcase," as in the usual classification of 2-bridge links (see, for example, $[\mathrm{HT}]$ ).

With the conventions that wrapping from front to back on the right edge corresponds to generator $a$, wrapping from back to front on the left edge corresponds to generator $b$, the lines have non-negative slope and are traversed from lower left to upper right on the front and the basepoint is located at the lowest intersection point of the lines with the left edge (see Figure 7), we read off $W(r, s, a, b)$ for the element in $\pi_{1}(H)$ corresponding to a line of slope $r / s$. Note that the lines may be taken to have non-negative slope less than 1 without loss of generality since increasing the slope by 1 has the effect of Dehn-twisting the pillowcase along the vertical bisector (which bounds a disc in $H$ ) and replacing $a$ by $a^{-1}$.

Thus, we may attach a singular 2-handle along an $r / s$ curve and obtain a 3dimensional orbifold (with boundary) whose orbifold fundamental group is the desired Generalized Triangle Group.

We should note here that a partial converse to this theorem was incorrectly 


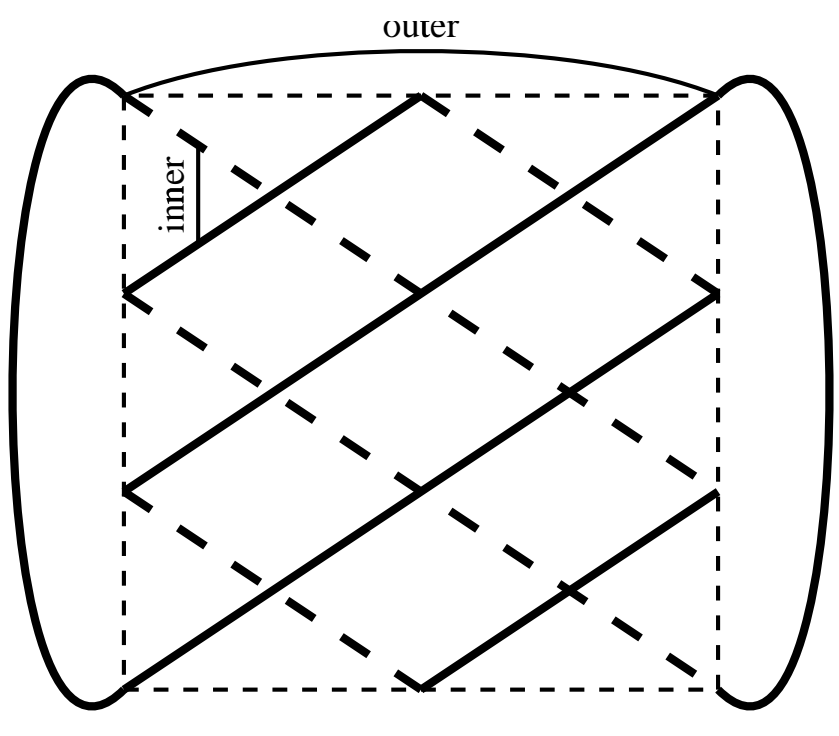

Figure 8

asserted in a previous version of this paper. We are indebted to Jim Howie for pointing this error out.

To make explicit the connection with $\S 2$ we argue as follows: let $G$ be a generalized triangle group as in Theorem 8 (i.e. $G=\Delta(m, n, p, W(r, s, a, b))$ for some integers $r, s, m, n, p)$. Then, $G$ is the orbifold fundamental group of a compact orientable 3-orbifold. As discussed in the proof of Theorem 8, we construct the orbifold by taking a genus 2 handlebody $H$ and attaching singular 2-handles along simple closed curves in the boundary of the handlebody representing $a, b$ and $W(r, s, a, b)$ where $a$ and $b$ are the standard generating curves in $\partial H$. Consider then, the 3 -manifold obtained by attaching a 2-handle to $H$ along the curve $W(r, s, a, b)$. Depending on whether the curve representing $W(r, s, a, b)$ is separating or not (which depends on whether $s$ is even or odd), we get a compact manifold $M$ with two or one torus boundary components, respectively. Viewing $M$ as a knot or link complement in a closed 3-manifold, we see that the two generator presentation for $\pi_{1}(M)$ arising from the above 2-handle attachment, yields generators which are meridians of the knot or link. By killing these meridians we see that $M$ is a knot or link complement in a homotopy 3 -sphere $\Sigma$.

One explicit way to realize this link complement in a homotopy 3 -sphere is with a 2-bridge link in a 3-sphere, as we will see below. In fact, this is the only way to realize this, since $\Sigma$ has a genus 2 Heegard splitting, and there are no Heegard-genus 2 counterexamples to the Poincare Conjecture (see [MB], for example). Furthermore, [BZ] implies that a link complement which is generated by two meridians must be a 2-bridge link.

To see precisely which unknottings of 2-bridge links correspond to which group, we will look a bit more carefully at the handlebody $H$ : we can see that the 2bridge link which is being unknotted is (equivalently) the $r / s$ rational link with the "inner" unknotting tunnel or the the $p / s$ rational link, where $p r \equiv 1(\bmod s)$, with the "outer" unknotting tunnel (see Figure 8). To see this, observe that the nontrivial curve which bounds a disc in a 3-ball with two unknotted arcs removed is the boundary of a regular neighborhood of the homotopic image (rel. endpoints) of either arc pushed to the boundary 2 -sphere. This is exactly the relation between 
the simple closed curve in Figure 6 and the two arcs in the standard 2-bridge picture (again, see Figure 8). So, the meridian of the unknotting tunnel corresponds to the "inner" unknotting in Figure 8. But, the usual classification of two-bridge links yields the fact that interchanging the inside and outside of a rational link diagram converts $r / s$ to $p / s$ where $p r \equiv 1(\bmod s)$.

We now recall some salient definitions from the theory of 3-orbifolds, cf. [Du1], [Sc] and [T1].

A compact 2-dimensional orbifold is said to be respectively spherical or toric if it is the quotient by a finite (smooth) group action of the 2 -sphere $S^{2}$ or the torus $T^{2}$. A 2-orbifold is called bad if it has no manifold cover. Following [Du2] we define a turnover to be a 2-orbifold with underlying space $S^{2}$ and three cone points, and a pillow orbifold to be a 2-orbifold with underlying space $S^{2}$ and having four cone points, all of which have cone angle $\pi$.

A compact orientable 3-orbifold $Q$ is said to be irreducible if it contains no bad 2suborbifold and every spherical 2-suborbifold bounds a BALL, that is, the quotient of the 3-ball by a finite group of orientation preserving isometries of $S^{2}$.

An embedded orientable non-spherical 2-orbifold $S$ in $Q$ is incompressible if any 1-suborbifold of $S$ which bounds a DISC (that is, a 2-orbifold of the form $\mathbb{D}^{2} / F$ where $F$ is a finite subgroup of $O(2))$ in $Q \backslash S$ bounds a DISC in $S$.

We define a 2-orbifold $S$ properly embedded in a 3 -orbifold $Q$ to be essential if it is not boundary parallel and $S$ is either spherical and does not bound a BALL or $S$ is non-spherical and is incompressible.

$Q$ is said to be simple if every incompressible toric suborbifold is boundary parallel.

The following conjecture of Thurston [T2] (see [Ho] for a discussion and outline of the proof) provides the existence of a geometric structure.

Thurston's Orbifold Conjecture. Let $Q$ be a compact irreducible orientable simple 3-orbifold possibly with boundary. If $\partial Q \neq \emptyset$ assume that it consists of Euclidean suborbifolds, and that the (nonempty) singular set has dimension 1 . Then $Q$ has a geometric structure.

Using this conjecture we shall establish:

Theorem 9. Let $Q(m, n, p)$ be a 3-dimensional orbifold with underlying space $S^{3}$ and singular set a 2-bridge knot or link L together with an unknotting tunnel, labelled $m, n$ and $p$, with $p$ along the unknotting tunnel (and possibly with vertex neighborhoods deleted as in §2). Let $r, s$ denote positive relatively prime integers with $r<s$ such that $\pi_{1}^{\text {orb }}(Q)=\Delta(m, n, p, W(r, s, a, b))$. Then $Q(m, n, p)$ is geometric unless $L$ is a link of two unknotted, unlinked components. More specifically,

(1) if $L$ is a link of two unknotted, unlinked components, then $Q$ is bad.

(2) otherwise, if $\pi_{1}^{\text {orb }}(Q)$ is finite, then $Q$ is spherical

(3) otherwise, if $s$ is odd, $p=2$, either $m$ or $n=2$, and $2 r \equiv \pm 1(\bmod s)$ then $Q$ is Seifert-fibered

(4) otherwise, if $r=0, s=1$ then $Q$ is Seifert-fibered (actually, I-fibered)

(5) otherwise, $Q$ is hyperbolic

Proof. Denote the singular set of $Q$ by $\Sigma$. We need to categorize the spherical and toric 2-orbifolds in $Q$. Note that there are no essential spheres or tori in $Q$ since the complement of $\Sigma$ is a handlebody. Nor are there any 2-orbifolds with boundary 
reflectors, since $Q$ is an orientable orbifold. Thus, we must deal with the 2 -orbifolds having underlying space $S^{2}$ and up to 4 cone points.

Let us examine carefully a few particular types of orbifolds we will need to recognize. All of these will have underlying space a 3-ball (possibly with some vertex neighborhoods deleted). We will refer to such an orbifold with singular set a single vertex and three unknotted singular arcs running out to the boundary as a "Y-ball," to an orbifold with one unknotted singular arc as an "I-ball," and to an orbifold with two unknotted singular arcs as an "II-ball" (see Figure 9). In the case of a Y-ball, the interior vertex will possibly have a neighborhood deleted if the incident edges are not consistent with the vertex having a spherical link.

We note that each one of these special orbifolds may be recognized up to isotopy by the combinatorial type of the singular set, together with the fundamental group of the complement of any subgraph of the singular set being free (see [ST]). We also observe that the boundary of any of these special orbifolds is inessential.

Let us call a 3-orbifold basic if it has underlying space a 3-ball, singular set combinatorially equivalent to one of the 3 special orbifolds in Figure 9, and if the fundamental group of the complement of any proper subgraph of the singular set is free.

Let us suppose a 2-orbifold $O$ in $Q$ bounds a basic 3-orbifold $B$. Then, either $B \backslash \Sigma$ has free fundamental group (in which case $B$ is one of the special orbifolds) or $B \backslash \Sigma$ fails to inject into $Q \backslash \Sigma$ (since $Q \backslash \Sigma$ is a handlebody) and thus some essential loop $\gamma$ in $O \backslash \Sigma$ bounds a disc in $Q \backslash(\Sigma \cup O)$. If $\gamma$ bounds a DISC in $O$, then we have a bad 2-orbifold in $Q$ by pasting the DISC and the disc together. Otherwise, $O$ is compressible in $Q$. Thus, any 2-orbifold in $Q$ that bounds a basic 3 -orbifold must either be inessential or give rise to a bad 2-orbifold in $Q$.
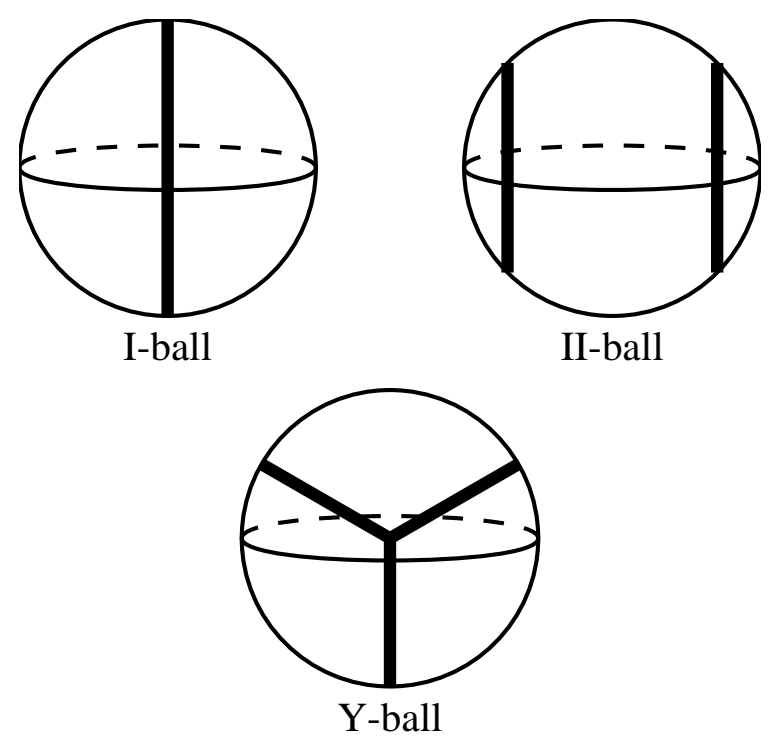

Figure 9

Let us next fix some notation: denote by $\tau$ the unknotting tunnel, and by $K$ the 2-bridge knot (if it is a knot) or by $L_{1}$ and $L_{2}$ the two components of the link. In the knot case, denote by $K_{1}$ and $K_{2}$ the two arcs of $K$ between the two intersections with $\tau$. Let $S$ be an embedded sphere in $Q$ meeting the singular set in $q$ points, where $1 \leq q \leq 4$. Observe that the $L_{i}$ are unknotted circles and the $K_{i}$ are unknotted arcs. We will show that $Q$ is geometric (unless $L$ is a 2-component 
unlink) by showing that $S$ can only be bad if $L$ is a 2-component unlink, and that otherwise $S$ bounds a basic orbifold.

Note that $q$ cannot be 1 , unless $L$ is a two component unlink, since no sphere can meet $L$ in a single point, and a sphere that meets only $\tau$ in an odd number of points must separate the two components of $L$ (and cannot occur at all if $L$ is a knot). So, except for the $Q$ arising from this particular link, there are no bad 2-suborbifolds in $Q$.

If $q=2$, then either $S$ meets $\tau$ twice or one of the $L_{i}$ twice or one of the $K_{i}$ twice. In either case, $S$ bounds a basic orbifold equivalent to an I-ball.

If $q=3$ (turnover), then $S$ must meet $\tau$ exactly once, and either some $L_{i}$ twice or both $K_{i}$ once: it cannot meet $L$ (or $K$ ) an odd number of times for homological reasons, and it cannot meet $\tau 3$ times as observed above. In the knot case, since 2-bridge knots are prime, one of the balls bounded by $S$ must contain an unknotted arc of $K$. This ball will also necessarily contain an unknotted subarc of $\tau$ and so it is a basic orbifold equivalent to a Y-ball. In the 2-component case, one of the balls bounded by $S$ will contain the other component of $L$ and the other one will not. The ball which does not contain the other component must contain only an unknotted arc of $L_{i}$ (since $L_{i}$ is in fact an unknot) and an unknotted arc of $\tau$ and so is also a basic orbifold equivalent to a Y-ball.

If $q=4$ (pillow), then there are several combinatorial possibilities, but in every case $S$ bounds two balls, one of which contains both vertices and the other of which contains none. The side containing no vertices is a basic orbifold equivalent to an II-ball.

This shows that $Q$ is geometric as long as there are no (deleted) vertices with link a hyperbolic 2-orbifold. If there are such vertices, we may perform the usual trick of doubling along the links of such vertices (denote this double by $D Q$ ), and observing that any essential toric or spherical 2-orbifolds in $D Q$ gives rise to an essential toric or spherical 2-orbifold inside one of the halves. This is because the doubling surfaces are 2-spheres with 3 cone points and any essential embedded 2-orbifold placed in general position will intersect the doubling surface at most in simple closed curves, which all bound a DISC in the doubling surface. Using an inductive process on innermost such curves, we may decompose the original 2-orbifold into an essential 2-orbifold in one half, together with some (possibly empty) collection of essential 2 -spheres with 2 cone points (again embedded in one of the halves). Thus, if there are no essential spherical or toric 2-orbifolds in each half, there are none in $D Q$.

So, $D Q$ must be geometric. Observe that since $\pi_{1}^{\text {orb }}(D Q)$ is infinite and contains a Fuchsian triangle group, the geometric structure on $D Q$ cannot be modelled on $S^{3}, S^{2} \times \mathbb{R}, E^{3}, N i l$ or Sol. Therefore either the double is hyperbolic or it is a

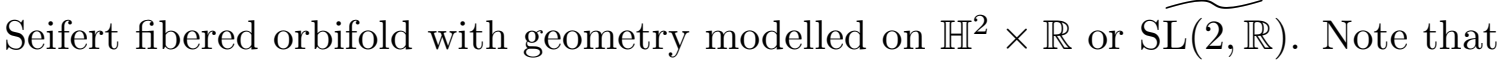
$D Q$ admits an orientation-reversing involution $\sigma_{0}$ interchanging the two copies of $Q$. As is well-known, in the hyperbolic case, we can make this involution an isometry, hence forcing $Q$ to be hyperbolic and making the boundary of $Q$ totally geodesic. In the Seifert fibered cases, since $\pi_{1}^{\text {orb }}(D Q)$ is residually finite, we can pass to a regular finite cover $D M$ of $D Q$ which is a manifold and which admits a lift $\sigma$ of the orientation reversing involution switching the two copies of $Q$. This induces a covering of $Q$ by a manifold. Thus we have a finite group $G$ acting on the geometric manifold $D M$. By Meeks and Scott [MS], since we are in the Seifert fibered case, the $G$-action preserves the geometric structure, and so in particular $\sigma$ acts as an 
isometry of the geometric structure on $D M$. Hence we deduce that the involution $\sigma_{0}$ on $D Q$ preserves the geometric structure and so we deduce that $Q$ is geometric.

In fact, the only essential hyperbolic 2-orbifolds that exist in a Seifert-fibered 3-orbifold are "horizontal" 2-orbifolds, which cut the 3-orbifold apart into an $I$ bundle. Since the doubling surfaces are 2-sided, the $I$-bundle must in fact be trivial, so this only occurs when $r=0, s=1$ (i.e., when the Generalized Triangle Group is actually a Triangle Group).

Now that $Q$ is geometric, to complete the proof we need to decide which geometry is possessed by $Q$. By the above comments we may restrict to the case in which the doubling argument above was not needed.

By Theorem 1 of [Du1] $Q$ must either be spherical (and have finite $\pi_{1}$ ), Seifertfibered (with either finite or infinite $\pi_{1}$ ), one of 12 Euclidean orbifolds (infinite $\pi_{1}$ ), a solvorbifold (infinite $\pi_{1}$ ) or a hyperbolic orbifold (infinite $\pi_{1}$ ). Note that Seifert-fibered orbifolds with finite $\pi_{1}$ are also spherical.

Now, we can rule out the 12 "special" Euclidean orbifolds by checking Dunbar's list directly. We rule out the Seifert-fibered case by observing that the only possible vertex stabilizers for Seifert-fibered orbifolds with infinite fundamental group are cyclic or dihedral (see $\S 4$ and $\S 5$ of [Du1]), and that the only possible $Q(m, n, p)$ which have vertex stabilizers of this form are the orbifolds $Q(2,2, p)$ and $Q(2, n, 2)$ (or $Q(m, 2,2)$ ). The former have finite (dihedral) fundamental group and thus are spherical, whether or not they are fibered.

The latter only arise in the 2-bridge knot case (the link case has vertex indices of $(2,2,2)$ and $(2, n, n))$. Assume for definiteness that $K_{1}$ is labelled 2. Observe that here, the 2-fold cyclic branched cover of $S^{3}$ branched over the unknot $\tau \cup K_{1}$ is again $S^{3}$ and that the union of the two lifts of $K_{2}$ is a knot $J$. Then $Q$ is Seifertfibered if and only if $J$ is a torus knot. We claim that this will happen precisely when $r \equiv \pm 1(\bmod s)$.

To see this, recall the geometric picture of $Q$ with its vertices deleted which was given in the proof of Theorem 2, i.e., a genus-two handlebody with singular discs attached along two standard meridians $a$ and $b$ and a simple closed curve representing $W(r, s, a, b)$. The 2 -fold branched cover corresponds to a genus-three handlebody (cover corresponding to index-2 subgroup freely generated by $a^{2}, b$, $a b a^{-1}$ ) with singular discs attached to the lifts of $b$ and $a b a^{-1}$ and nonsingular discs attached to the lifts of $a^{2}$ and $W(r, s, a, b)^{2}$. Now, note that if $a$ has order 2 , $W(r, s, a, b)^{2}=W\left(2 r, s, a b a^{-1}, b\right)$, so that this orbifold is a punctured realization of the group $\Delta\left(n, n, 1, W(2 r, s, c, d)\right.$ ) (where $c$ and $d$ are lifts of $a b a^{-1}$ and $b$, respectively). In particular, $J$ is a 2-bridge knot and is a torus knot if and only if $2 r \equiv \pm 1$ $(\bmod s)$.

We rule out the solvorbifold case by recalling Theorem 1 of [Du1] which shows that solvorbifolds fiber over $S^{1}$ or have a double cover which does. The abelianization of $\pi_{1}^{\text {orb }}(Q)$ is clearly finite (since both generators have finite order), so $Q$ itself doesn't fiber over $S^{1}$. Furthermore, if there exist any index-2 subgroups, they are generated by $\left\{a^{2}, b, a b a^{-1}\right\},\left\{b^{2}, a, b a b^{-1}\right\}$ or $\left\{a^{2}, b a^{-1}, a b\right\}$. The first two of these also have obviously finite abelianization. To handle the third subgroup (which only occurs when $m$ and $n$ are both even and either $p$ is even or $L$ is a link), let $A=a^{2}$, $B=b a^{-1}$ and $C=a b$. Then, in the abelianization, $A, B+C$ and $B-C$ all have finite order and hence so does $2 B$ and hence $B$. Thus, the generating set $\{A, B, B C\}$ is a set of generators which all have finite-order in the abelianization.

To see that no cusped orbifolds (those with vertex links that are Euclidean 
triangle groups) result in Seifert-fibered (or I-fibered, which we will henceforth not distinguish) orbifolds, other than the cases mentioned in the theorem, recall that totally geodesic surfaces in Seifert-fibered spaces are either transverse to the fibers (in which case they decompose the space into a union of I-bundles) or are parallel to the fibers (in which case they are fibered themselves).

Now, suppose that $Q$ is a Seifert-fibered orbifold with either one or two cusps. If either cusp is transverse, then $Q$ is an I-bundle (so the other cusp if it exists must be transverse also). If there are two cusps (note that this always occurs if the 2-bridge link is a knot), then the I-bundle must be trivial and we are in case (4) of the conclusion of the theorem. If there is only one cusp, then the other vertex must be dihedral (as observed earlier) and so the singular locus must consist of a singular fiber connecting the cusp to itself, another loop of 2-fold singularity connected to the cusp by the unknotting tunnel. But, this cannot be an I-bundle, since taking the 2-fold cyclic cover over the singular loop should produce another I-bundle, but instead produces a two-cusped orbifold with one singular arc joining the two cusps and a singular arc running from each cusp to itself (note that the singular arcs are fibers of the I-bundle).

If no cusps are transverse, then there must be a "parallel" cusp (that is, a cusp that is itself fibered). Such a cusp must have only 2-fold singularities and so must be a 2 -sphere with four 2 -fold cone points (pillow cusp), but all the cusps in $Q$ are 3 -cone point (turnover) cusps.

We note here that the proof of Theorem 9 also yields the fact that all elements in these Generalized Triangle Groups that appear to have finite order, actually do have the order that the presentation would imply (this was used in the proof of Lemma 4 in $\S 2$ ). The only way for this to fail in a 3-orbifold is for the orbifold to contain a bad 2-suborbifold, and our analysis rules out such suborbifolds.

\section{REFERENCES}

[AR] C. Adams and A. W. Reid, Unknotting tunnels in two-bridge knot and link complements, To appear Comment. Math. Helv..

[BMS] G.Baumslag, J. W. Morgan and P. B. Shalen, Generalized triangle groups, Math. Proc. Camb. Phil. Soc. 102 (1987), 25-31.

[BM] S. Bleiler and Y. Moriah, Heegaard splittings and branched coverings of $B^{3}$, Math. Annalen 281 (1988), 531-543.

[BZ] M. Boileau and B. Zimmerman, The $\pi$-orbifold group of a link, Math. Zeit. 200 (1989), $187-208$.

[CFJR] T. Chinburg, E. Friedman, K. N. Jones and A. W. Reid, The Smallest Volume Arithmetic Hyperbolic 3-Manifold, In preparation.

[Du1] W. Dunbar, Geometric orbifolds, Revista Mat. Univ. Complutense 1 (1988), 67-99.

[Du2] W. Dunbar, Hierarchies for 3-orbifolds, Topology and its Appl. 29 (1988), 267-283.

[DM] W. Dunbar and G. R. Meyerhoff, Volumes of hyperbolic 3-orbifolds, Indiana J. Math. 43 (1994), 611-637.

[G93] A. Duncan, N. Gilbert, J. Howie, eds., Combinatorial and Geometric Group Theory, Edinburgh 1993, L.M.S. Lecture Note Series 204,, Cambridge University Press, 1995.

[EEK] A. L. Edmonds, J. H. Ewing and R. S. Kulkarni, Torsion free subgroups of Fuchsian groups and tessellations of surfaces, Invent. Math. 69 (1982), 331-346.

[FF] B. Fine and C. Frohman, Some amalgam structures for Bianchi groups, Proc. A. M. S. 102 (1988), 221-229.

[GL] C. McA. Gordon and J. Luecke, Only integral Dehn surgeries can yield reducible manifolds, Math. Proc. Camb. Phil. Soc. 102 (1987), 97-101. 
[H] M. Hagelberg, Generalized triangle groups and 3-dimensional orbifolds, SFB 343 Bielefeld, Diskrete Strukturen in der Mathematik. Preprint 92-049 (1992).

[HMR] M. Hagelberg, C. Maclachlan and G. Rosenberger, On discrete generalized triangle groups, Proc. Edinburgh Math. Soc. 38 (1995), 397-412.

[HT] A. Hatcher and W. P. Thurston, Incompressible surfaces in 2-bridge knot complements, Invent. Math. 79 (1985), 225-246.

[HMV] H. Helling, J. Mennicke and E. B. Vinberg, On some general triangle groups and 3dimensional orbifolds, To appear Trans. Moscow Math. Soc.

[Ho] C. D. Hodgson, Geometric stuctures on 3-dimensional orbifolds: Notes on Thurston's Proof, Preprint.

[LR] F. Levin and G. Rosenberger, A class of SQ-universal groups, In Group Theory, Proceedings Of The 1987 Conference, Singapore (1989), De Gruyter Verlag.

[MB] J. W. Morgan and H. Bass, The Smith Conjecture, Academic Press, 1984.

[MS] W. H. Meeks III and G. P. Scott, Finite group actions on 3-manifolds, Invent. Math. 86 (1986), 287-346.

[Sc] G. P. Scott, The geometries of 3-manifolds, Bull. London Math. Soc. 15 (1983), 401-487.

[ST] M. Scharlemann and A. Thompson, Detecting unknotted graphs in 3-space, J. Differential Geometry 34 (1991), 539-560.

[T1] W. P. Thurston, The Geometry and Topology of Three-Manifolds, Mimeographed Lecture Notes, Princeton Univ., 1977.

[T2] W. P. Thurston, Three manifolds with symmetry, Preprint.

Dept. of Math. Sci., Ball State University, Muncie, IN 47306, U.S.A.

E-mail address: kerryj@math.bsu.edu

Dept. of Math., University of Texas, Austin, TX 78712, U.S.A.

E-mail address: areid@math.utexas.edu 